\title{
Représentation discursive des pairs et ethos dans les arrêts de la Cour de cassation française
}

\author{
Laurence CHAPUIS \\ Université Rey Juan Carlos \\ Département de Langues modernes \\ laurence.chapuis@urjc.es
}

Recibido: 28/09/2012

Aceptado: 19/12/2012

\begin{abstract}
Résumé
La Cour de cassation française se situe au sommet de la hiérarchie judiciaire. C'est l'organisme chargé de vérifier la conformité des décisions au droit. Son ethos préalable est dominant mais cette image sociale d'elle-même ne la soustrait pas à l'obligation de motiver ses arrêts, stratégiquement composés pour la lecture à l'audience et leur postérieure publication. Depuis une perspective discursive, l'on montre que l'argumentation véhiculée par les arrêts se fonde notamment sur l'attribution des rôles qui imprègne les relations entre les pairs et la Cour. L'inscription cadencée des instances obéit à des rites discursifs qui sont autant de traces d'une situation d'énonciation marquée sous le sceau du rituel judiciaire. La manifestation des rapports aux pairs, ainsi que la mise en scène rigide dans laquelle elle s'exerce, nous renseignent sur l'image discursive de la Cour, son ethos, dont la construction participe également de l'entreprise de persuasion.
\end{abstract}

Mots clés: Analyse du discours, argumentation, discours judiciaire, ethos.

\section{Representación de los pares en el discurso de la Cour de cassation francesa}

\section{Resumen}

La Cour de cassation de Francia se sitúa en la cúspide de la jerarquía judicial. Es el órgano responsable de verificar la conformidad de las decisiones a la ley. Su ethos previo es dominante, pero esta imagen social no la exime de argumentar sus sentencias, estratégicamente compuestas con vista a ser leídas para la audiencia y publicadas posteriormente. Desde una perspectiva discursiva, mostramos que la argumentación vehiculada por las sentencias se basa, entre otros, en la asignación de roles que determina la relación entre los jueces y la Cour. La inscripción en cadencia de los órganos judiciales obedece a ritos discursivos, reflejo de una situación de enunciación marcada por la impronta del ritual judicial. La manifestación de las relaciones con los jueces así como la rígida puesta en escena que conlleva, nos informan sobre la imagen discursiva de la Cour, su ethos, cuya construcción participa también de la estrategia de persuasión.

Palabras clave: análisis del discurso, argumentación, el discurso judicial, ethos. 


\title{
Representation of other judicial organs in French Cour de cassation discourse
}

\begin{abstract}
The French Cour de cassation is located at the apex of the judicial pyramid. The court is responsible for verifying the conformity of decisions to the law. Despite the dominance of this pre-discursive ethos, the organ is not exempt from justifying its decisions, which are strategically made to be read at the hearing, and then published. From a discursive approach, it will be shown that argumentation in those judicial decisions is based on the distribution of roles, which influences the relationships between the Cour and the judges. The discursive representation of judicial organs reveals a discursive routine linked to the situation of enunciation marked by the judicial ritual. The representation of relationships and rigid staging give us information about the discursive image of the Cour and its ethos, which is particularly relevant for its persuasive strategy.
\end{abstract}

Keywords: discourse analysis, argumentation, judicial discourse, ethos.

\section{Referencia normalizada}

Chapuis, L. (2013). "Représentation discursive des pairs et ethos dans les arrêts de la Cour de cassation française". Thélème, Vol. 28, 73-89.

\section{Introduction}

La Cour de cassation, institution de droit privé, est définie comme étant « la Cour suprême » dans le sens où elle occupe le sommet de la hiérarchie judiciaire française. Ses fonctions, cependant, sont bien différentes de celles attribuées aux tribunaux et Cours d'appel car elle ne tranche pas sur des questions de fait mais sur des erreurs de droit. Cornu (2007 : 244-245) en propose la définition suivante : il s'agit de la « juridiction la plus élevée de l'ordre judiciaire comprenant six chambres (cinq chambres civiles et une chambre criminelle) dont la mission est de veiller au respect de la loi en cassant les décisions en dernier ressort qui la violent et de faire régner l'unité d'interprétation du Droit». C'est l'organisme qui est chargé de vérifier la conformité des décisions judiciaires au droit. Elle rend ses décisions sous forme de réponse au pourvoi ${ }^{1}$ formulé par les demandeurs, au sein duquel ceux-ci dénoncent, de façon argumentée, l'illégalité d'un arrêt déterminé. Si la rédaction des arrêts est assumée par un juge-rapporteur, désigné spécialement pour mener à bien les recherches concernant l'affaire, la décision finale est prise par un collège de juges, rattaché

\footnotetext{
${ }^{1}$ Recours formé par le demandeur devant la Cour de cassation et contre une décision de justice rendue en dernier ressort (Cornu, 2004 : 687).
} 
à une chambre déterminée suivant les spécificités du droit concerné. Et le locuteur du discours est bel et bien la Cour, c'est elle qui s'en responsabilise, qui s'impose en dépassant la personnalité et les circonstances de chacun des magistrats qui la composent.

Sa position au faîte du système judiciaire — et l'ethos préalable qui en résulten'affranchit pas la Cour de ses obligations discursives : elle doit motiver ses arrêts. Les motifs, c'est-à-dire la justification de la décision de la Cour, prennent principalement place dans les arrêts de rejet ou de cassation ${ }^{2}$ dont l'organisation canonique, si peu souple et archi réglementée, institue une routine discursive profondément liée au rituel judiciaire. Ces types de sentence sont rattachés, pour reprendre la distinction de Maingueneau aux genres institués de mode 1, " peu sujets à variation » (Maingueneau, 2007 : 32). Le locuteur y détermine des rôles pour chacun et établit des rapports discursifs déterminants pour l'argumentation, et spécifiquement, pour la construction de sa propre image, son ethos (Amossy, 2006 : 79). La Cour organise stratégiquement son discours autour de trois instances principales : le demandeur qui sollicite l'avis de la Cour, l'auditoire universel qui reconnaît la portée de ses décisions et les juges qui ont rendu l'arrêt soumis à examen. Situant ces réflexions dans le cadre posé par l'argumentation dans le discours (Amossy, 2010 : 41-42), nous examinerons les rôles donnés par le locuteur aux instances concernées par l'affaire et la nature des relations qui en découlent ; laissant volontairement de côté les figures du dema

ndeur et de l'auditoire universel, nous nous centrerons sur les rapports entretenus avec les pairs. En dernier lieu, nous montrerons comment les rôles sont tenus en fonction d'une mise en scène discursive chargée de la symbolique judiciaire. Tout ceci nous éclairera sur l'ethos d'autorité que la Cour refaçonne discursivement, tant dans son modelage du rapport aux pairs, que dans sa gestion du rituel.

\section{Les rôles discursifs}

Afin de pouvoir déterminer comment la Cour représente son auditoire dans ses arrêts, il nous faut brièvement montrer comment le conflit monte jusqu'à elle. Lorsqu'une affaire tombe entre les mains de la justice, elle est d'abord jugée par les tribunaux du premier degré. Si la ou les parties en cause ne sont pas d'accord avec le jugement rendu, l'affaire peut être rejugée par une cour d'appel, située au deuxième degré des juridictions. L'appel constitue une voie de recours ordinaire, c'est un droit du plaideur (à ce stade, spécifiquement nommé «appelant» ou "partie appelante »). Les cours d'appel sont, autant que les tribunaux, des juges du droit et du fait. Elles reprennent donc les décisions dans leur intégralité, elles rejugent les

\footnotetext{
${ }^{2}$ L'arrêt de rejet rejette le pourvoi formé par le demandeur ; l'arrêt de cassation a une issue contraire : il casse la décision formulée par la juridiction contre laquelle est effectué le pourvoi.
} 
affaires dont elles sont saisies. Mais en cas de nouveau désaccord, les parties ne peuvent se pouvoir en cassation que si elles invoquent un vice de forme ou une violation de la loi. Si les cours d'appel constituent un deuxième degré de juridiction, la Cour de Cassation n'en est donc pas un troisième. Lorsqu'elle est saisie par un pourvoi, elle abandonne les questions de fait à l'appréciation des juges du fond car sa fonction est de veiller à la correcte application de la règle juridique (Guillien et al., $2001: 165)$.

Le discours de la Cour est régi par des consignes génériques qui varient légèrement suivant les branches du droit impliquées. La nature de l'affaire détermine celle de la chambre vers laquelle elle sera orientée. L'attribution à une chambre s'effectue dès l'origine quand la Cour se déclare compétente pour résoudre le problème de droit qui lui est posé ${ }^{3}$, en fonction de la matière et de la branche de droit concernées. La mise en relief de la classification thématique est conservée pour la publication. En effet, la Cour affiche sur son site très visiblement cette distinction par chambre, en regroupant au sein de bulletins détachés les arrêts des première, deuxième, troisième chambres civiles, de la chambre commerciale, économique et financière, de la chambre sociale et de la chambre criminelle.

La Cour traite ses pairs strictement dans le cadre de leurs fonctions ${ }^{4}$. Dans la pratique judiciaire, le locuteur et destinataires sont considérés sous une certaine facette dont la nature infléchit l'échange: "parler de rôle, c'est insister sur le fait que chaque genre de discours implique les partenaires à travers un statut déterminé, non dans toutes leurs déterminations possibles » (Maingueneau, 2009: 48). Pour reprendre l'exemple parlant de Maingueneau, les juges enrôlés dans l'affaire s'y impliquent en tant que tels et non en tant que père ou mère de $\mathrm{X}$ enfants. De la même manière, la Cour adresse sa réponse à un pourvoi rédigé par un «demandeur » exclusivement considéré comme tel. Il est attribué, aux individus ou sociétés engagés dans une voie judiciaire, un "rôle institutionnel joué » pour l'occasion (Cali, 2001 : 12). Et si certaines fois, l'action du demandeur implique qu'une autre partie soit assignée à comparaître, celle-ci adopte alors le rôle du " défendeur ». Une fois mise en route la machine juridique, les individus sont étiquetés et l'on observe un va et vient entre plusieurs désignations usées anaphoriquement qui facilitent la progression discursive et argumentative.

\footnotetext{
${ }^{3}$ Dans le but que le nombre croissant de pourvois ne nuise pas à la qualité de ses décisions, la Cour se livre, pour chaque pourvoi soumis, à un examen d'admissibilité afin de vérifier s'il est recevable, si les moyens sont sérieux, etc. Lorsque les conditions ne sont pas respectées, le pourvoi est déclaré non-admis.

${ }^{4}$ Les fonctions des magistrats judiciaires découlent de leur statut qui leur a été conféré par un attribut fondamental : ils ont obtenu leur diplôme à l'issue de l'École nationale de la magistrature et font partie d'un corps professionnel unique. Ils sont nommés par le Conseil Supérieur de la Magistrature, sont inamovibles et n'ont pas de contrôle hiérarchique direct, ce qui leur donne l'indépendance nécessaire pour formuler librement leurs décisions.
} 
La Cour inscrit donc dans son discours les principaux magistrats chargés de la résolution du problème, le rôle joué, posé en italiques, précédant le patronyme: «Président: M. Joly, conseiller doyen faisant fonction ; Rapporteur: Mme Degorce» (arrêt $\mathrm{n}^{\circ}$ 07-87.734: 950). Mais elle peut les désigner également dans la trame de l'interaction relatée; là aussi le statut éclipse presque toujours le nom ${ }^{5}$ des magistrats mentionnés: «l'appartenance au corps doit primer sur l'équation personnelle, la fonction sur le sujet » (Faget, 2008 : 9). Y apparaissent, dans ce cas, les destinataires-acteurs qui ont joué leur rôle en amont de la procédure : la Cour informe que «le juge d'instruction a saisi le juge des libertés et de la détention » (arrêt $n^{\circ} 08-84.893$ ), que « le procureur de la République avise [...] le juge des tutelles » (arrêt $\left.\mathrm{n}^{\circ} 10-83.283\right)$; il est question de «l'intervention d'un juge judiciaire » (arrêt $\left.\mathrm{n}^{\circ} 10-90.099\right)$, du «mémoire produit par l'avocat général » (arrêt $\mathrm{n}^{\mathrm{o}} 10-81.245$ ), de la "présence du ministère public » (arrêt $\mathrm{n}^{\circ} 09-84.108$ ), etc. Souvent, afin de ne pas se perdre dans le dédalle des appellations, elle use d'expressions simples, qui permettent de les situer dans la série ascendante de l'organisation judiciaire : "les premiers juges» qui reprend anaphoriquement n'importe quel tribunal et «les juges du second degré » (arrêt nº7-87.900: 834), qui, de manière identique reprend la Cour d'appel. Cette recatégorisation des instances permet de facilement situer le destinataire dans la procédure; c'est une manière simple de voir qui à dit quoi, et dans quel ordre (les premiers juges intervenant avant ceux du deuxième degré). Comme une décision de justice « intervient toujours à moment donné, dans un environnement donné [...], il faut avoir constamment à l'esprit les interactions entre les différentes phases et composantes du processus judiciaire » (Jean, $2007: 30$ ), vigilance d'autant plus exigée du fait de la potentielle variabilité des rôles joués par les parties tout au long du chemin parcouru par l'affaire. En effet, le «demandeur» qui a saisi le juge en première instance forme une demande contre un « défendeur »; s'il perd le procès et décide de faire appel en deuxième instance, il aura le rôle d' " appelant »; mais il sera « intimé » si c'est le défendeur qui est « appelant », c'est-à-dire si c'est ce dernier qui formule le recours.

Par ailleurs, nous observons qu'avant 2008, la Cour avait souvent tendance à employer une expression généralisante : «les juges du fond» qui renvoyait aux juges des tribunaux de l'ordre judiciaire et aux cours d'appel. Mais l'on remarque actuellement que cette expression n'est plus présente et que la Cour lui préfère des substituts comme « les juges », " ces tribunaux », « la cour d'appel »... Si la Cour ne fait pas référence à l'individu considéré par son statut, elle élargit son propos à

\footnotetext{
${ }^{5}$ Leur patronyme n'apparaît que très rarement et uniquement lorsque les circonstances l'exigent. Dans les arrêts considérés pour l'étude, il n'y a qu'une inscription de deux noms et elle se doit au fait que cette donnée était déterminante pour le verdict : "attendu que l'arrêt mentionne qu'il a été signé par Mme Fontaine, présidente, et lu par M. Potée, conseiller ; que ces mentions établissent la régularité de la décision $[\ldots] »\left(\operatorname{arrêt~}^{\circ} 08-80.204: 851\right)$.
} 
l'institution. Très souvent, elle précise la désignation avec une indication toponymique : «le juge d'instruction de Paris a, en exécution d'une demande d'entraide judiciaire présentée par le procureur près le Tribunal pénal international pour le Rwanda » (arrêt $\left.n^{\circ} 07-85.108: 856\right)$; ou encore « le procureur général près la Cour de Lyon a interjeté l'appel de ce jugement » (arrêt n $\mathrm{n}^{\circ} 08-80.598: 858$ ). Dès le départ, l'institution est mise en relation avec un lieu (les juridictions sont toujours attachées aux toponymes) mais la pratique les unit à travers un complément du nom et non pas par un complément circonstanciel: il s'agit de «la cour d'appel d'Aix-enProvence» (arrêt $\mathrm{n}^{0} 08-80.872$ ) ou de «la cour d'appel de Paris, $11^{\mathrm{e}}$ chambre » (arrêt $n^{\circ} 07-82.249: 883$ ). L'usage des toponymes permet d'identifier les lieux liés aux juridictions qui ont participé à l'élaboration de la décision attaquée, et d'ainsi récapituler une partie ou l'intégralité du chemin parcouru par l'affaire. Par ailleurs, la multiplicité des juridictions nommées rappelle les limites de l'étendue de leurs compétences territoriales et souligne, par contraste, l'unicité de la Cour et son rayonnement extensif partout en France.

\section{La relation aux pairs}

\subsection{La Cour inscrit son discours dans une culture juridique commune}

Tout en respectant la distance de rigueur, la Cour exhibe les signes de l'expérience, de la sagesse et d'une faculté d'analyse et de jugements communes, liés à l'appartenance d'une même culture. Toutes les instances sont parfaitement hiérarchisées mais elles partagent la culture de l'écrit et un certain savoir. Les décisions font donc partie d'une «culture graphique séculaire et sophistiquée » (Fraenkel, 2006:30), avec un usage de la langue et un raisonnement particuliers se coulant dans le moule de la tradition.

La Cour présente son discours comme le fruit d'un travail pyramidal, s'écrivant « à plusieurs mains » (Fraenkel, $2006: 81$ ). En amont de la sentence, les recherches qui sont effectuées pour la prise de décision juste, révèlent un travail en réseau qui inclut la participation du personnel d'appui. La décision englobe non seulement les discours des parties identifiées et des autres juges, de manière à la fois explicite et implicite, mais elle retient également le travail des greffes et du service de documentation de la Cour. La tradition juridique française donnant toujours une grande importance à l'écrit ${ }^{6}$, la chaîne discursive juridique est constituée, à ce niveau

\footnotetext{
${ }^{6}$ «Le droit est une discipline traditionnellement liée à l'écrit. Les mots sont, et restent, les signes privilégiés du travail herméneutique du juriste» (Bourcier \& Mackay, 1992). Dans le cas des arrêts, cette affirmation est ici d'autant plus appropriée que le « pourvoi en cassation doit être dirigé contre un jugement », c'est-à-dire que la loi oblige le pourvoi à se former à partir d'un texte officiel. En effet, " on ne peut se pourvoir que contre une décision, non contre un simple avis précédant la décision, cette dernière faisant seule véritablement grief » (Boré, $1985: 32$ ).
} 
juridictionnel, de nombreux maillons clairement référencés qui consistent en une multitude de documents aux fonctions diverses (qui prouvent, attestent, certifient...). Ils se contredisent parfois, se complètent en d'autres occasions et c'est au collège des juges instruits de trancher, à partir de la confrontation des discours, après les autres instances judiciaires et devant toute la communauté juridique, et même la société. Au bout de la procédure, toutes les entreprises se fondent en une ultime décision qui sera lue aux parties et publiée au bulletin. Ainsi, le discours de l'arrêt retrace les faits de procédures utiles à la décision et propose souvent un résumé succinct qui retrace le cheminement de l'affaire ${ }^{7}$ : « attendu qu'il résulte de l'arrêt attaqué et des pièces de procédure que Richard X [...] et Philippe Y...[...] ont été poursuivis devant le tribunal correctionnel [...] ; que les juges du premier degré ont condamné les prévenus ; attendu qu'en l'état de ces seuls motifs [...] la cour d'appel a fait l'exacte application de la loi » $\left(\mathrm{n}^{\circ} 07-81.661: 809\right)$. D'une part, comme nous l'avons dit, la Cour soutient son discours sur ceux des juridictions antérieures ${ }^{8}$, dont l'ordre temporel d'occurrence est marqué et sans lesquels elle ne pourrait émettre le sien. Sans conflit graphiquement marqué, validé, discuté, sans autres décisions, sans antériorité scripturale, la Cour ne peut se prononcer. Toutes les parties engagées doivent garder en tête " plusieurs sources énonciatives distinctes et concomitantes » (Fraenkel, $2006: 12$ ) qui transcrivent une réalité complexe, matérialisée et fixée par la tradition de l'écrit. D'autre part, la désignation claire des autres juridictions renvoie au concept de "bonne justice", comme le définit Jean-Luc Aubert (2004 : 168). En fait, la mise en évidence de l'organisation aussi parcellaire des instances est en cohérence avec le principe de séparation des pouvoirs tel qu'il est pensé dans la conception classique de la démocratie issue des lumières, et selon laquelle aucun organe de la société ne doit tous les concentrer?.

\subsection{La Cour évalue, supervise et corrige ses pairs}

Les juges qui ont rendu la décision attaquée sont soucieux du résultat du pourvoi : « ces juges, même dans leur souveraineté, ne sont pas à l'abri d'un regard, toujours bienveillant, mais qui peut être aussi sévère, de la Cour régulatrice» (Buffet, 2004 : 118). Le dispositif ${ }^{10}$ les intègre également explicitement. Ainsi, quand le pourvoi est rejeté, c'est que « la cour d'appel a justifié sa décision »; et lorsque l'arrêt est cassé,

\footnotetext{
${ }^{7}$ Cette démarche est particulièrement évidente dans les arrêts de rejet.

${ }^{8}$ De la même manière, les autres juridictions fondent leurs discours sur d'autres écrits mis en forme pour servir d'appui à leurs décisions : " la France entière, si l'on accepte de la regarder ainsi, produit inlassablement et, pour ainsi dire, sécrète par tous ses pores des documents innombrables qui peuvent se transformer d'un seul coup, si les circonstances l'exigent, en preuves utilisables pour une affaire » (Latour, $2002: 88$ ).

${ }^{9}$ La liberté des citoyens résulte de cette séparation des pouvoirs de faire la loi (législatif), de la faire appliquer (exécutif) et d'en sanctionner les manquements (judiciaire), afin que le pouvoir arrête le pouvoir.

${ }^{10}$ Partie de l'arrêt qui intervient après les motifs et inclut la décision des juges.
} 
c'est parce que « la cour d'appel a méconnu le texte susvisé »" ${ }^{11}$. En d'autres termes, si la solution est le rejet, c'est parce que la Cour considère que la règle de droit a été correctement appliquée : l'affaire est classée et la juridiction émettrice obtient son épaulement tacite. A l'inverse, si la Cour casse l'arrêt, c'est parce qu'une erreur a été commise et elle indique dans quel sens il faudrait rejuger l'affaire. Elle la renvoie alors à une autre juridiction de fond, ou à la même que celle dont la décision a été cassée mais dans une formation différente, comme il est, par exemple indiqué dans de dispositif de 1'arrêt n08-82-434 : "CASSE ET ANNULE, en toutes ses dispositions, l'arrêt susvisé de la Cour d'appel de Metz [...], et pour qu'il soit à nouveau jugé, conformément à la loi ; RENVOIE la cause et les parties devant la cour d'appel de Metz, autrement composée ». Si l'une des qualités des juges est la vigilance $^{12}$, on peut aisément imaginer qu'ils ne perdent l'affaire de vue que lorsque le rejet est prononcé ; car si c'est la cassation que décide la Cour, alors le procès n'est pas terminé et l'on considère qu'ils constituent un destinataire aux abois, guettant le moment de reprendre ou, au contraire, de fermer définitivement le dossier. En effet, ils ont été rappelés à l'ordre sur un point qui est précisé, et qu'ils doivent reprendre et examiner afin de ne plus se tromper ${ }^{13}$. L'arrêt présuppose donc des juges attentifs à l'exercice de contrôle de la Cour et disposés à se plier à ses indications dont ils savent qu'elles sont le fruit d'une étude approfondie. Notons que seuls les juges les plus expérimentés (comme ceux de la cour d'appel de Paris ou d'Aix-en-Provence) se risquent à contester 1'orientation posée par la Cour; les autres, en suivant son avis, reconnaissent son autorité indiscutable.

Les juges sont conscients d'être traités avec justesse car la Cour reconnaît toujours leur parcelle de savoir et de compétence. Elle n'énonce en effet pas le fait dans ces arrêts et montre bien que, sauf exception, les juges du fond sont souverains dans leurs constatations ${ }^{14}$. Il y a donc, de la part du juge de cassation, une reconnaissance du travail des juges antérieurs qui ont étiqueté juridiquement les faits exposés par le justiciable: « cette traduction se fait par un incessant va-et-vient du fait au droit, et non par une qualification simultanée. Le juge confronte chacun des éléments de fait à la règle de droit pour découvrir si la qualification qu'il a pressentie est justifiée en chacun des aspects » (Robin, 2000 : 342). Les juges du fond repris par le rapporteur procèdent à des « reconstructions » que celui-ci ne remet pas en question. Ces faits-là sont donc posés, dans les arrêts, comme le savoir commun du locuteur et de co-énonciateurs. La Cour se voit parfois dans l'obligation

\footnotetext{
${ }^{11}$ Ce sont là des formulations les plus courantes.

${ }^{12}$ Bien avant, en rédigeant leur jugement, les juges du fond s'adressent déjà au juge potentiel qui pourrait exercer son contrôle (Cornu $2000: 349$ ).

${ }^{13}$ La cassation peut également être rendue «sans renvoi », ce qui signifie que la Cour décide de mettre elle-même fin au litige (Cornu, 2004 : 788).

${ }^{14}$ L'un des fondements de l'argumentation des juges de la Cour de cassation repose sur les faits qui sont connus des destinataires les plus directs. Afin de bien juger, celle-ci doit maîtriser les faits qui ont été à l'origine de la décision attaquée et ils ne sont pas remis en question (Cornu, $2000: 342$ ).
} 
de le rappeler, elle parle alors d' «appréciation souveraine, par les juges du fond, des faits et circonstances de la cause » (arrêt $n^{\circ} 07-88.699: 819$ ). Toutefois, une certaine orientation est toujours donnée dans la narration des faits car la Cour, comme toutes les autres juridictions, active sa recherche en fonction de problèmes qui sont soulevés par le demandeur et qui l'obligent à formuler des choix: « le juge n'est pas là pour revenir sur les évènements passés mais seulement sur ceux qui sont nécessaires à la succession » (Doat, $2006: 404$ ).

En outre, parfois, lorsque l'argumentation le requiert, la Cour exprime littéralement la rigueur du personnel de justice tout au long de la procédure :

Que le procureur de la république, après avoir fait procéder à une enquête préliminaire sur ces faits, a requis l'ouverture d'une nouvelle information, contre personne non dénommée, pour blanchiment d'argent [...], escroqueries, abus de confiance, recel, faux et usage de faux ; que le juge d'instruction, saisi de l'information initiale, a été désigné pour instruire également sur ces faits ; qu'il a versé au dossier de la procédure des copies de pièces issues de la première information et délivré une commission rogatoire en exécution de laquelle Marc $\mathrm{X}$ a été placé en garde à vue, le 27 mars 2007, et entendu à plusieurs reprises (arrêt nº8-83.064:947).

Cette énumération des tâches scrupuleusement réalisées par les magistrats a pour vertu de montrer le travail abattu par tout le personnel de justice qui s'organise de façon cohérente, sur la base d'actions toutes certifiées par les discours. Le locuteur met en relief la méticulosité d'un travail dont il est laissé trace. La progression des magistrats s'effectue avec une rigueur tant juridique que linguistique et dans la conscience que tout doit être reporté.

Mais la Cour n'est pas une simple observatrice. Elle évalue le savoir-faire et les comportements professionnels des juges qui ont rendu la décision. La Cour qui valide ou sanctionne une combinaison d'application de connaissances (qu'elle détermine quand elle affirme que tels juges ont fait « l'exacte application de la loi ») exerce son rôle correcteur, formateur et éducateur. Le sémantisme des verbes introducteurs est révélateur du rôle de la Cour en tant qu'évaluatrice. François Weber (2009), dans une communication parue sur le site de la Cour de cassation, apporte des éclaircissements sur le contenu sémantique de certains termes. Dans les arrêts de rejet, on observe dans les structures argumentatives l'usage de verbes introducteurs du discours indirect dont les nuances sont soulignées par ce juriste. Ainsi, selon lui, «énonce que » (arrêt $n^{\circ} 11-84.180: 55 /$ arrêt no $08-88.426: 644$ ), implique la reproduction exacte des termes de la décision attaquée et n'apporte aucune précision sur le contrôle. "Constate que " $\left(\mathrm{n}^{\circ} 10-85.678: 3\right)$, correspond à une appréciation souveraine des faits par les juges du fond. «Relève que » (arrêt n $\mathrm{n}^{\circ} 08-83.759$ : 1146) porte sur des considérations et circonstance de fait tandis que « retient que » (arrêt $\mathrm{n}^{\circ} 11-84.694: 72 /$ arrêt $\mathrm{n}^{\circ} 10-85.678: 2 /$ arrêt $\mathrm{n}^{\circ} 11.87 .688: 144$ ), correspond à une appréciation de faits ayant une incidence d'ordre juridique. Pour les initiés, chacun de ces verbes revêtit un sens précis alors que dans le langage courant les différences sémantiques sont nettement moins marquées.

Au prix d'un labeur sans relâche, la Cour guide donc les autres juges dans la recherche de la perfection. En matière civile, dans l'extension de sa mission de cor- 
rection, la Cour de cassation a même prévu des fiches méthodologiques sur l'intranet ${ }^{15}$ pour éviter certains vices de forme et réduire l'afflux des décisions, ce qui permettrait à ses juges d'accorder une plus grande attention aux pourvois qui soulèvent des points plus épineux : «elles [les fiches méthodologiques] répondent au souci de faciliter aux juges d'appel l'exercice de leur mission, et de prévenir des erreurs ou des irrégularités de forme ou de fond propres à susciter des pourvois en cassation » (Bulletin d'information $\mathrm{n}^{\circ} 613$ du 15/02/2005). Ces échanges avec les juges du fond montrent bien la conscience des juges de la Cour des « dysfonctionnements de l'institution judiciaire dans son ensemble » (Weber, 2006: 125) et leur volonté d'y remédier. Tous ces discours qui aident à l'amélioration de la rédaction des décisions mettent en évidence l'importance d'une bonne formulation des arrêts reposant sur des capacités juridiques et une domination de l'exercice discursif.

Les cours d'appel ou autres tribunaux visés dans le discours de la Cour sont mis à distance, de la même manière que le sont les parties : « le juge du fond est libre dans ses appréciations, mais c'est une liberté surveillée » (Buffet, 2004 : 119). La Cour opère systématiquement des vérifications qui sont mises en évidence par un lien consistant avec les textes fondateurs. Tout est calibré, contrasté et s'effectue sous de multiples conditions contenues dans la loi : « dès lors que la qualité de la justice dépend du bon déroulement des procédures, il apparait en effet indispensable de se préoccuper, de manière générale, des conditions dans lesquelles la justice est administrée et rendue » (Frydman, 2007 : 25). Le maintien de l'éloignement de la Cour est la condition pour que s'exerce librement son contrôle. L'examen des données qui lui sont soumises exige un détachement manifeste qui se traduit par l'emploi de la passivation et des formes impersonnelles. C'est souvent de cette manière que la Cour met le doigt sur un attribut, une obligation qui n'ont pas été respectés ou dont l'application a été contestée ${ }^{16}:$ " la cour d'appel, à laquelle il incombait de renvoyer ... » (arrêt $\left.n^{\circ} 08-80-489: 876\right)$; « les juges [...] étaient tenus [...] de faire droit à la demande » (arrêt $\left.\mathrm{n}^{\circ} 07-88.699: 821\right)$. La Cour fait également, à leur égard, un usage pragmatique de la restriction. L'emploi de "ne...que " introduit les erreurs soulignées, en limitant le champ de réalisation de la proposition concernant les capacités des juges concernés: «le juge d'instruction a la faculté de ne mettre en examen une personne déterminée qu'après s'être éclairé [...] sur sa participation aux agissements incriminés » (arrêt $\left.n^{\circ} 08-83.064\right)$. La Cour pratique aussi la restriction dans sa forme adjectivale («seul») qui souligne l'exclusivité d'une fonction, d'un aspect juridique, etc. : " le juge d'instruction, seul habilité à l'aviser de...» (arrêt nº8-82.924: 796), « seul un officier de police [...]

\footnotetext{
${ }_{16}^{15} \mathrm{http}$ //intranet.cour-de-cassation.intranet.justice.fr/Rpvjcc/Methodologie/Form.htm.

${ }^{16}$ Comme on l'a vu, la Cour rehausse son rôle de correcteur dans les remarques des décisions de cassation. Le rejet les conforte dans la qualité de leur décision. La Cour marque une certaine approbation à travers des formules telles que « ... a fait l'exacte application de la loi ». Elle peut remarquer aussi que la Cour «a, sans insuffisance ni contradiction, répondu... » (arrêt nº7-87.207 : 819), etc.
} 
peut procéder à 》 (arrêt $\mathrm{n}^{\circ} 10-82.699$ : 609). Par ailleurs, le motif à la cassation est toujours inscrit dans le paragraphe qui précède le dispositif. Le connecteur " mais » est l'outil par excellence de la Cour lorsqu'elle repousse la décision des juges du fond et guide le destinataire vers la cassation. «Mais » apparait toujours dans un mouvement argumentatif propre à ce résultat du pourvoi. Il marque la réfutation de l'argument présenté dans le contexte gauche et casse l'arrêt attaqué en précisant l'erreur commise et apportant ainsi une solution possible :

Attendu que [...] l'arrêt énonce, en se fondant sur les seules pièces annexées par le procureur de la République à son réquisitoire, que les propos de Laurence X..., visés dans la plainte, ont été tenus alors qu'elle était entendue en qualité de témoin assisté dans une procédure distincte [...]; que les juges en concluent que ces propos ne peuvent, en l'absence de spontanéité, être constitutifs d'une dénonciation calomnieuse ni recevoir aucune qualification pénale ;

Mais attendu qu'en prononçant ainsi, en l'absence de tout acte propre à l'affaire en cause et sans avoir vérifié par une information préalable et contradictoire la réalité des faits dénoncés dans la plainte, la chambre de l'instruction a méconnu les textes susvisés (arrêt nº $08-88.426: 644$ ).

Attendu que, pour déclarer irrecevables les constitutions des parties civiles de la Société protectrice des animaux de la Fondation Brigitte Bardot, l'arrêt confirmatif attaqué relève que les statuts des deux associations ne précisent pas que leur président, qui les représente dans tous les actes civils, soit habilité à agir en justice, et ajoute que ce dernier ne dispose pas d'un mandat exprès ;

Mais attendu qu'en prononçant ainsi, sans rechercher si les statuts de chacune des associations appelantes, qui autorisent le président à la représenter en justice, n'emportaient pas pour ce dernier, en l'absence de stipulations contraires, le pouvoir d'agir en justice, la cour d'appel n'a pas justifié sa décision (arrêt n $\left.n^{\circ} 08-83.759: 1146\right)$.

Attendu que tout jugement ou arrêt doit comporter les motifs propres à justifier la décision et répondre aux articulations essentielles des mémoires des parties ; que l'insuffisance ou la contradiction des motifs équivaut à leur absence ;

Attendu que, pour confirmer l'ordonnance de placement en détention provisoire de Pascal X..., l'arrêt attaqué prononce par les motifs reproduits au moyen ;

Mais attendu qu'en se déterminant ainsi, sans répondre au mémoire dans lequel la personne mise en examen faisait valoir que son état de santé était incompatible avec une mesure de détention provisoire, le tribunal supérieur d'appel n'a pas justifié sa décision (arrêt nº9-84.172: 656).

L'on ne peut manquer de relever la préposition «sans » qui marque à chaque fois ce qui a fait défaut ; appliquée ici aux verbes « vérifier », "rechercher », « répondre », elle sous-entend le non-accomplissement de tâches dont la réalisation semble pourtant inhérente à l'exercice habituel de leur fonction de juger. La Cour dénonce que l'action des juges visés s'écarte du bon sens requis pour l'exercice habituel de la profession. La juridiction de renvoi devra donc porter remède à l'erreur commise, en effectuant l'action marquée par l'infinitif inséré dans le groupe prépositionnel. L'on remarque que la Cour peut également pondérer l'effet de « sans » :

Attendu qu'il résulte de l'arrêt attaqué que, interpellé à $10 \mathrm{~h} 15$ et retenu ensuite contre son gré, X... n'a été informé que 3 heures plus tard des raisons pour lesquelles il était privé de liberté ;

Attendu que, pour estimer que n'avaient pas été méconnues les dispositions de l'article 5, paragraphe 2, de la Convention européenne des droits de l'homme, selon lesquelles toute personne arrêtée doit être informée, dans le plus court délai et dans une langue qu'elle comprend, des raisons 
de son arrestation et de toute accusation portée contre elle, la chambre d'accusation relève que ce retard se justifie par les particularités de l'espèce ;

Mais attendu qu'en se déterminant ainsi, sans mieux s'expliquer sur les raisons [...], la chambre d'accusation n'a pas donné de base légale à sa décision (arrêt nº8-86.791:5).

La préposition «sans » est ici appliquée au terme conséquent «s'expliquer » dont le sens est nuancé par l'adverbe " mieux ». La Cour relève que la besogne ne s'est effectuée que partiellement et incite à l'observation d'une grande minutie. Elle se définit ainsi précise et opiniâtre.

L'élévation de la Cour, qui promène méticuleusement son regard au-dessus de la mêlée, renseigne sur les rapports de subordination avec les locuteurs de l'arrêt critiqué. Mais cette distance empreinte d'impartialité, s'évanouit parfois sous l'emprise de la sévérité. La Cour se montre neutre, certes, mais elle ne se cache pas de rappeler les juges du fond à l'ordre lorsqu'il lui paraît qu'ils se sont mépris grandement. Ainsi dans l'arrêt de rejet $n^{\circ} 98-80.048$, dans lequel elle donne en définitive raison aux juges du fond, elle sent que la règle de droit a été en partie piétinée et le mécontentement pointe sous l'aspect glacial de la remontrance: « attendu qu'en prononçant ainsi [...], la chambre d'accusation a fait une fausse application des dispositions conventionnelles précitées; attendu que, cependant, abstraction faite des énonciations erronées mais surabondantes ${ }^{17}$ relatives à ces dispositions, l'arrêt attaqué n'encourt pas la censure ». La critique, dans ce cas, se greffe sur le terme "fausses» qui s'applique à une action de la chambre d'accusation non conforme au droit. Puis elle s'exerce à travers une formule (« erroné mais surabondant ») qui, bien que standardisée et ne faisant guère de sens que dans les énoncés juridiques, laisse affleurer la réprimande. Elle fait remarquer aux juges que les motifs ainsi qualifiés n'ont rien apporté au raisonnement qui soutient leur décision ${ }^{18}$. Même si la décision n'encourt pas la cassation, la Cour invite les juges à redoubler de rigueur. L'acidité de la critique se prolonge ici pour souligner les lacunes à combler.

La position discursive de la Cour au-dessus de celle de ses pairs met en relief l'existence d'une hiérarchie indispensable au bon fonctionnement de la justice. Mais la relation aux autres juges rehausse aussi les traits d'identité communs, liés à une connaissance profonde du droit. Dans les décisions, l'on remarque bien que l'appartenance au microcosme judiciaire est également porté par l'attachement à

\footnotetext{
${ }^{17}$ « Surabondant» désigne, dans le cas des motifs un «élément inutile (et souvent critiquable) de la motivation d'une décision de justice, qui, bien qu'erroné, n'empêche pas cette décision d'être maintenue (si elle est par ailleurs suffisamment étayée), dès lors que la juridiction de contrôle peut en faire abstraction » (Cornu, $2004: 882$ ).

${ }^{18} \mathrm{La}$ Cour ici ne se contredit évidemment pas. Elle met juste en exergue que la décision reste juste en dépit des erreurs soulignées : "les motifs critiqués ne sont pas le seul fondement de la décision attaquée qui peut être sauvée par un autre motif non contesté » (Weber 2009: 8).
} 
des rites extensibles aux diverses juridictions et à toutes leurs activités. La Cour se pose comme la garante du rituel judiciaire dont la solennité jaillit dans le texte.

\section{Mise en scène discursive}

L'élaboration de l'arrêt est régie par de lourdes contraintes déterminant la distribution des rôles, le format, les pratiques langagières, etc. Cela est dû aux particularités de la situation d'énonciation qui se greffent immanquablement dans le texte: comme le prononcé de la décision s'effectue obligatoirement en audience publique, l'arrêt porte et perpétue les traces du montage solennel qui s'effectue dans les lieux consacrés de la Cour. Du fait des analogies qui ont été détectées entre le discours judiciaire et le discours théâtral ${ }^{19}$, nous avons choisi de parler de « mise en scène », expression jugée plus à même de refléter le concept de rituel qui se joue dans la situation d'énonciation de ces discours. Le décor, le port des costumes judiciaires, les tours de paroles, etc. aident à la représentation du conflit au palais de justice qui permettra de pacifier la situation.

Le lien social entre les individus ayant été rompu du fait de leurs divergences, la justice lui substitue le lien juridique. Dans le corps de l'arrêt s'effectue la représentation de la situation d'énonciation. Locuteur et destinataires sont tenus de jouer leur rôle eu égard à un script qui ne tolère pas l'improvisation. Dans le cadre du droit civil, l'interprétation des personnages vise à la réparation du dommage (Moor, 1999) ; en droit pénal, elle prétend sanctionner les infractions ou les crimes, punir le coupable et le dissuader de recommencer.

Si l'arrêt admet peu de variantes, c'est parce qu'il doit être le gage d'une bonne justice. Celui-ci postule, entre autres et comme tout discours, un sens dans la communication: « la parole dans un genre de discours ne va pas, en effet, de n'importe qui vers n'importe qui » (Maingueneau, 2009: 42). C'est la Cour de cassation qui est saisie par un demandeur; dans le cadre du genre, son intervention surgit d'un fait précis et parce que son opinion a été sollicitée ; ce n'est donc pas elle qui établit le premier rapport de communication et celui-ci n'aurait pas lieu s'il n'y avait la démarche préalable d'un premier locuteur, en l'occurrence le demandeur. Le discours du demandeur est cité dans la décision en donnant une priorité au sens : sa question précède textuellement la réponse de la Cour. Mais c'est la Cour qui rend la

\footnotetext{
${ }^{19}$ « Il y a en effet dans le théâtre comme dans la justice des personnages (sujets de droit) derrière les personnes, qui interprètent sur la scène du droit un rôle conféré et réglé par un texte auquel ils sont tenus, (re)jouant une représentation du conflit entre demandeur et défendeur, entre victime et bourreau, mis en scène par une procédure, dans des costumes et un décor rituels (robes et Palais), devant des spectateurs, en respectant les trois règles du théâtre classique de l'unité de lieu (salle d'audience), de temps (le procès se joue d'un seul trait de son ouverture au jugement) et d'action (une seule action est jugée), ses trois coups (marteau ou sonnette, lever des spectateurs, procession des juges - dramatisation de l'entrée en scène du juge)» (Flückiger, $2001: 4$ ).
} 
décision et elle surplombe le discours en six lettres majuscules : LA COUR. Si l'usage veut que la Cour se reconnaisse à sa seule majuscule (puisque les cours d'appel n'en prennent pas), on ne s'étonne pas d'en trouver tant au début de la décision car l'exclusivité de la Haute-juridiction passe, à notre avis, par l'utilisation d'une typographie déterminée. L'exagération majusculaire aurait ici deux fonctions complémentaires, motivées par la mise en rapport avec le temps et le lieu sacrés de l'audience. D'abord, elles opèrent une mise en contexte car le détachement typographique acquiert une équivalence acoustique avec le heurt du marteau ou le tintement de la sonnette. Elles signalent donc l'entrée des magistrats revêtus de leurs habits distinctifs marquant, par le seul habillement, la séparation entre la justice et les êtres du monde. Resitués, ces mots agissent comme de véritables déictiques, ils sont l'appel de l'huissier qui «prévient de l'entrée des magistrats dans la salle d'audience et amène le public à se lever et se plonger dans un silence solennel » (Desprez, 2009 : 2). Et la deuxième fonction est que cette emphase, loin de traduire une obséquiosité quelconque, témoigne du prestige de l'institution et du caractère sacré de l'action de la justice. Elles rappellent cette ligne de démarcation entre la justice et les citoyens et affichent l'autorité de la Cour. À l'écrit, la Cour assied d'abord sa souveraineté sur des majuscules.

Le discours judiciaire est transmis publiquement à l'audience, qui constitue de ce fait la première scène d'énonciation. Mais il est destiné, par sa publication postérieure, à être élevé au dessus de sa première situation; c'est en cela qu'il tend à reproduire, toutes les fois qui seront nécessaires, la mise en scène de la justice et le moment précis où elle s'exerce. La décision de la Cour lue à l'audience est donc non seulement marquée par le lieu, mais aussi par le temps qui s'arrête, se mue et se sacralise : «pendant toute la durée de l'audience, le temps judiciaire dans le lieu de justice, accompagné de gestes, formules, vêtements et règles procédurales spécifiques, marque une rupture avec le monde environnant » (Desprez, 2009: 65). La lecture de la décision est concrètement vue comme une transition, comme un passage entre le temps sacré de l'audience et le temps de la vie réelle: "le président, en énonçant la décision à la personne mise en cause debout, puis en prononçant la fin de l'audience, permet aux acteurs du procès et au public de revenir dans l'écoulement quotidien du temps » (Desprez, 2009: 65). Les majuscules du verdict dans «CASSE » ou «REJETTE » marquent la fin du procès tout comme la COUR en annonçait le commencement. Le procès est donc circonscrit à l'écrit par une amplification typographique : l'arrêt se clôt avec le dispositif, la boucle se ferme. C'est en cela qu' " on ne peut dissocier les normes d'organisation des discours et les normes d'organisation des hommes» (Maingueneau, $2005: 3$ ).

Par ailleurs, tout au long de la décision, il y a une constante résonnance entre le discours et les images représentatives de la justice. Le langage aride et solennel véhicule, en très peu de mots, une symbolique séculaire. La partie de l'arrêt destinée à la Cour est très brève, mais le laconisme, ici, augmente le degré de lisibilité du discours car il sauve les mots de la perte de sens qu'un bavardage excessif ne manquerait de provoquer. Les pairs comprennent rapidement la solution proposée à la querelle. La cadence des connecteurs («alors que », «attendu que») équilibre 
visiblement le discours ; aussi bien les arguments du pourvoi que ceux avancés dans la décision attaquée par les juges antérieurs sont calibrés par la Cour et cette évaluation a sa traduction symbolique dans la balance ${ }^{20}$ qui pèse les arguments. L'équilibre dans le discours véhicule le concept d'équité. Il montre comment, par l'action de la justice, les conflits s'apaisent et rappelle la fonction de juger qui doit départager les parties en évaluant tous les moyens présentés. Le rétablissement par le discours judiciaire de l'équilibre entre les parties - l'égalité du citoyen devant la loi- compense l'asymétrie des relations sociales qui s'exerce à l'extérieur des tribunaux. La justice s'énonce comme mesure et comme sagesse. Les actes de langage finaux («casse» et « rejette») sont, quant à eux, en connexion avec le glaive ${ }^{21}$ qui tranche. Ils imposent l'exécution de la décision. La Cour achève son discours en rappelant qu'elle est le locuteur responsable du dire et que c'est son discours qui est en mesure de modifier le réel. Et lorsqu'elle ajoute, dans la lignée pragmatique de ce qui précède, « renvoie » et « dit», elle agit directement sur ses pairs en leur retournant une affaire qu'ils devront juger en fonction de ses consignes.

\section{Conclusion}

Les rôles et la mise en scène discursive prolongent l'ethos préalable de la Cour. Son exigence pointue alimente sa suprématie. À travers sa relation aux pairs, elle met en évidence des liens de savoir et de pratiques qui renforcent la cohésion de la communauté des juges. Cette manœuvre permet aux plus rétifs d'accepter les rappels à l'ordre de la Cour et de corriger efficacement les erreurs désignées. En montrant qu'elle parvient à donner à la loi un sens adapté à chaque cas particulier, elle met encore en évidence son engagement pour la meilleure justice possible. Enfin, les rites d'écriture par lesquels la Cour témoigne de son attachement au rituel judiciaire, indispensable au bon fonctionnement des institutions, parachèvent cette image : l'autorité résonne dans le discours, indubitable et persuasive.

\section{RÉFÉRENCES BIBLIOGRAPHIQUES}

Amossy, R., (2006) L'argumentation dans le discours. Paris, Armand Colin. Amossy, R., (2010) La présentation de soi. Ethos et identité verbale. Paris, Puf. Aubert, J.-L., (2004) Introduction au droit. Paris, Armand Colin. Boré, J., (1985) La cassation en matière pénale. Paris, Puf.

\footnotetext{
${ }^{20}$ L'une des plus anciennes représentations de la justice est la balance : la symbolique judiciaire utilise, par exemple, depuis le XIIIe siècle une figure de la mythologie grecque Thémis, déesse aux yeux bandés évoquant l'équité.

${ }^{21}$ Cet autre symbole, le glaive rappelle que la justice est liée à la force découlant de l'autorité de l'institution, sans laquelle il ne serait envisageable d'appliquer les décisions.
} 
Bourcier, D. \& P. Mackay, (1992) Lire le droit, Langue, texte, cognition. Paris, Librairie Générale de Droit et de Jurisprudence.

Buffet, J., (2004) "Le contrôle de la Cour de cassation et le pouvoir souverain" in Molfessis, N. (dir.), La Cour de cassation et l'élaboration du droit. Paris, Economica, pp.113- 124.

Cali, Ch., (2001) "Les échanges rituels dans les conférences internationales" in Les Carnets $d u$ Cediscor [En ligne]. $\mathrm{N}^{\circ} 7$, disponible sur: http://cediscor.revues.org/311 [Dernier accès le 18 juillet 2012]

Cornu, G., (2007) Vocabulaire juridique. Paris, Puf.

Desprez, F., (2009) Rituel judiciaire et procès pénal. Paris, L.G.D.J Lextenso Editions.

Doat, M., (2006) "Le jugement comme récit" in L'office du juge [En ligne]. Paris, Archives des colloques du Palais du Luxembourg, pp.396-416, disponible sur: http://www.senat.fr/colloques/office_du_juge.pdf [Dernier accès le 18 juillet 2012].

Faget, J., (2008) "La fabrique de la décision pénale. Une dialectique des asservissements et des émancipations" in Champ pénal / Penal field, nouvelle revue internationale de criminologie [En ligne]. Vol. V, disponible sur: http://champpenal.revues.org/3983 [Dernier accès le 18 juillet 2012].

Flückiger, A., (2001) "L'acteur et le droit : du comédien au stratège" in Revue européenne des sciences sociales [En ligne]. NXXXIX-121, disponible sur: http://ress.revues.org/645 [Dernier accès le 18 juillet 2012].

Fraenkel, B., (2006) "Actes écrits, actes oraux: la performativité à l'épreuve de l'écriture" in Études de communication [En ligne]. №29/2006, disponible sur: http://edc.revues.org/index369.html [Dernier accès le 24 août 2012].

Frydman, B., (2007) "L'évolution des critères et des modes de contrôle de la qualité des décisions de justice" in Mbongo, P. (dir), La qualité des décisions de justice. Strasbourg, Éditions du Conseil de l'Europe, pp. 18-29.

Guillien, R et al., (2001) Lexique des termes juridiques. Paris, Dalloz.

Jean, J-P., (2007) "La qualité des décisions de justice au sens du Conseil de l'Europe" in Mbongo, P. (dir), La qualité des décisions de justice. Strasbourg, Éditions du Conseil de l'Europe, pp. 30-48.

Maingueneau, D., (2005) "Détachement et surdestinataire. La correspondance entre Pascal et les Roannez" in Semen [En ligne]. N ${ }^{\circ} 20$, disponible sur: http://semen.revues.org/1086 [Dernier accès le 18 juillet 2012].

Maingueneau, D., (2007) "Genres de discours et modes de généricité" in Le français aujourd'hui. $\mathrm{N}^{\mathrm{o}} 159$, pp. 29-35.

Maingueneau, D., (2009) Analyser les textes de communication. Paris, Armind Colin, $2^{\mathrm{e}}$ édition.

Moor, P., (1999) "Le droit comme mise en scène" in Revue européenne des sciences sociales. Tome XXXVII, Nº113, pp.129-147.

Robin, C., (2000) La langue du procès. Clermont-Ferrand, Les Presses Universitaires de la Faculté de Droit de Clermont-Ferrand.

Weber, F., (2006) La Cour de cassation. Paris, La Documentation française. 
Weber, F., (2009) "Fiche méthodologique: comprendre un arrêt de la Cour de cassation rendu en matière civile" in Bulletin d'information $n^{\circ} 702$ du 15 mai [En ligne]. Disponible sur: http://www.courdecassation.fr/publications_cour_26/ bulletin_information_cour_cassation_27 [Dernier accès le 12 mars 2013]. 